\title{
UM SIMULADOR DINÂMICO DO CRESCIMENTO DE UMA CULTURA DE CANA-DE-AÇÚCAR
}

\author{
ANTONIO ROBERTO PEREIRA $\left({ }^{2}\right)$ e EDUARDO CARUSO MACHADO $\left({ }^{3,4}\right)$
}

\begin{abstract}
RESUMO
Este trabalho descreve a primeira versão de um simula dor matemático-fisiológico do crescimento diário de uma cultura de cana-de-açúcar (SIMCANA) em resposta às condições do ambiente durante a estação de crescimento. SIMCANA resume a maior parte das informações disponíveis concernentes aos processos fisiológicos da cultura de cana-de-açúcar. Esta sua versão não inclui os processos de igerminação e florescimento, havendo necessidade de especificar as condiçōes da cultura no primeiro dia de simulação. Em função das condições diárias de radiação solar global, temperatura máxima e minima, umidade relativa do ar, SIMCANA calcula as taxas de fotossíntese, respiração e crescimento da cultura, as taxas de senescência das folhas e raízes, a massa seca das folhas, colmos e raízes, e o índice de área foliar. Embora várias relações empiricas tenham sido usadas, SIMCANA parece ser capaz de simular o crescimento da cultura de cana-de-açúcar.
\end{abstract}

Termos de indexação: simulação; modelo matemático-fisiologico; fotossíntese, taxa; respiração, taxa; crescimento, taxa; área foliar, índice; cana-de-açúcar. pinas, SP.

$\left({ }^{1}\right)$ Recebido para publicação em 25 de maio de 1985 .

$\left({ }^{2}\right)$ Seção de Climatologia Agrícola, Instituto Agronômico (IAC), Caixa Postal 28, 13001 Cam-

$\left({ }^{3}\right)$ Seção de Fisiologia, IAC.

$\left({ }^{4}\right)$ Com bolsa de suplementação do CNPq. 


\section{INTRODUÇĀO}

Com o advento da crise mundial de energia, a cana-de-açúcar emergiu como principal fonte alternativa renovável, justificando a intensificação de pesquisas visando conhecer melhor o comportamento fisiológico dessa cultura através da análise quantitativa do crescimento em condições naturais. A elaboração de um modelo para simular tal crescimento evoluiu da necessidade de integrar os conhec imentos disponíveis, bem como prever o comportamento da cultura sob condições naturais.

Embora a cana-de-açúcar tenha assumido importância econômica desde os primórdios da civilização humana, ocupando cerca de 12 milhões de hectares em 79 países, em latitudes variando de $36^{\circ} \mathrm{N}$. até $31^{\circ} \mathrm{S}$. (IRVINE, 1983), apenas uma única tentativa de elaboração de um modelo específico para essa cultura (BULL \& TOVEY, 1974) está registrada na literatura.

A tentativa de elaboração de um modelo matemático-fisiológico oferece, segundo THORNLEY (1976), uma série de vantagens, pois: (a) informações a respeito de diferentes processos fisiológicos podem ser reunidas em um único modelo, para se ter idéia da cultura como um todo; (b) um modelo resume convenientemente grande quantidade de informações; (c) a base matemática para as hipóteses adotadas permite compreender quantitativamente a natureza das interações ambiente-planta; (d) a modelagem estimula novas idéias; (e) a elaboração de um modelo ajuda a detectar áreas onde o conhec imento é limitado; (f) modelos permitem interpolações e previsões.

O presente trabalho descreve a primeira versão de um simulador matemático-fisiológico do acúmulo de matéria seca de uma cultura de cana-de-açúcar (SIMCANA), desenvolvido com base no conhecimento das interações entre os processos fisiológicos e o ambiente.

\section{FORMULAÇÃO DE SIMCANA}

SIMCANA consiste em uma série de equações que descrevem quantitativamente o comportamento fisiológico da cultura em resposta à variação temporal das condições ambientes. Não foi intenção descrever detalhadamente os processos fisiológicos, mas procurar simular adequadamente o acúmulo de matéria seca.

O presente modelo é o mais simples possível. Descreve uma condição média da cultura e não de plantas individuais, e considera que: (a) as plantas estão no estádio vegetativo, ou seja, o processo de germinação não é incluído; (b) a cultura não apresenta deficiência nutricional; e, (c) a cultura está livre de infestações de pragas e doenças. A presente versão não inclui o processo de florescimento, devido à falta de informações específicas, embora as condi- 
ções necessárias à indução ao florescimento sejam conhecidas (PEREIRA et alii, 1983). Os parâmetros e constantes das equações que compõem o modelo foram obtidos com base na adaptação de resultados disponíve is na literatura. dada por:

A massa seca da cultura (PCUL $-\mathrm{g} \cdot \mathrm{m}^{-2}$ ) num dado instante t é

$$
\operatorname{PCUL}(t)=\operatorname{PCUL}(t-1)+\operatorname{TCCUL}(t) * \operatorname{DELTAT},
$$

onde TCCUL é a taxa de crescimento da cultura $\left(\mathrm{g} \cdot \mathrm{m}^{-2} \cdot \mathrm{d}^{-1}\right)$, e DELTAT, o intervalo de tempo considerado (1 dia). A taxa de crescimento da cultura representa o balanço entre a taxa de produção através da fotossíntese e a taxa de perda através da respiração.

Matematicamente, TCCUL é dada por THORNLEY (1976), McCREE \& SILSBURY (1978) e WIT et alii (1979):

$$
\text { TCCUL }=\text { EFIC * (FOTCUL }-M * \text { PCUL), }
$$

onde FOTCUL é a taxa de fotossíntese bruta da cultura $\left(g . \mathrm{m}^{-2} \cdot \mathrm{d}^{-1}\right)$, PCUL é a massa seca da cultura (g. $\left.\mathrm{m}^{-2}\right)$, e os parâmetros EFIC e $M$ representam, respectivamente, a eficiência de conversão de material fotossintetizado em massa seca e o coeficiente de manutenção dos processos fisiológicos da cultura (McCREE, 1970; THORNLEY, 1970).

A eficiência de conversão de material fotossintetizado, EFIC, varia entre 0,70 e 0,80 (HUNT \& LOOMIS, 1979), dependendo da composição química da fitomassa formada (PENNING de VRIES, 1975a), não sendo afetada pelas condições do ambiente (McCREE \& SILSBURY, 1978; McCREE \& KRESOVICH, 1978; WILSON et alii, 1980). Utilizando a composição química média da cana-de-açúcar (VALSECHI \& OLIVEIRA, 1964), adotou-se, no presente modelo, EFIC $=0,79$, ou seja, é necessário $1,27 \mathrm{~g}$ de glicose para formar $1 \mathrm{~g}$ de fitomassa.

\subsection{Taxa de fotossintese - FOTCUL}

O ganho de matéria seca (MS) pela cultura se dá exclusivamente através da fotossíntese. A estimativa da taxa diária de fotossíntese pela cultura FOTCUL (gMS.m $\mathrm{m}^{-2} \cdot \mathrm{d}^{-1}$ ), primeiro termo da equação (2), foi feita através do produto:

$$
\text { FOTCUL }=\text { FOTMAX * IAF * FLUZ * Fi, }
$$

onde FOTMAX representa a taxa máxima possivel para a cultura se as condições de ambiente forem ideais; IAF é o índice de área foliar da cultura e representa o tamanho do aparelho fotossintetizante naquele dia; FLUZ é a razão entre os comprimentos do dia e da noite; e Fi representa fatores de ajustamento, que variam entre 0,0 e 1,0 e que corrigem a taxa de fotossíntese da cultura para níveis reais compat íveis com as condições reinantesdurante 
o dia considerado. Os fatores de ajustamento representam uma estimativa do efeito de cada elemento do clima ou estádio de desenvolvimento da cultura sobre a taxa de fotossíntese (JAGER, 1974; JAGER \& KING, 1974; HOLT et alii, 1975; HODGES et alii, 1979). O produto FOTMAX * IAF * * FLUZ representa a taxa potencial de fotossíntese da cultura em dado dia.

O fator fotoperíodo (FLUZ) torna a taxa de fotossíntese potencial da cultura função da época do ano e do local considerado, sendo calculado através da expressão:

$$
F L U Z=\text { FOTOPE/(24 - FOTOPE). }
$$

O comprimento do dia (FOTOPE), em horas, é dado por

$$
\begin{aligned}
& \text { FOTOPE }=0,133 *(180 / \pi) *\left(\pi / 2-\arctan \left(X /(1-X)^{0,5}\right)\right) \\
& X=-\tan (A L A T I) * \tan (D E C L I N A C ̣ A ̄ O) \\
& \text { DECLINAC̣ÃO }=-23,45 *(\pi / 180) * \cos (2 \pi *(D I A+10) / 365),(7)
\end{aligned}
$$

onde DIA é número do dia no ano contado a partir de 1 9 de janeiro, e ALATI é a latitude local, em graus (GOUDRIAAN \& LAAR, 1978).

\subsubsection{Fatores de ajustamento - Fi}

Fator radiação solar (FRAD) - Assumiu-se que a taxa de fotossíntese da cultura em função da radiação solar (RAD) exibe, como a taxa de fotossíntese de uma folha isolada (GOUDRIAAN \& LAAR, 1978), uma curva de resposta do tipo Michaelis-Menten tendendo a um nível de saturação ao redor de $700 \mathrm{ly}$. Adaptando-se os valores de fotossintese medidos por BULL $(1969,1971)$ em folhas individuais de plantas jovens de cana-de-açúcar, est imou-se FRAD através da relação

$$
\text { FRAD }=(1-r) * \operatorname{RAD} /(355+0,5 *(1-r) * \operatorname{RAD}) \text {, }
$$

onde RAD é o total diário de radiação solar global, em ly. $\mathrm{d}^{-1}$, e r, o coeficiente de reflexão da radiação global. MACHADO et alii (1985) encontraram $r=0,2$ para a variedade NA56-79.

Fator idade da cultura (FIDADE) - Segundo vários autores (HARTT \& BURR, 1965; KORTSCHAK \& FORBES, 1969; BULL, 1969, 1971), a taxa de fotossíntese decresce acentuadamente com a idade da planta. Adaptando os resultados de HARTT \& BURR (1965), obteve-se

$$
\text { FIDADE }=\operatorname{EXP}(0,08812-0,89104 * \text { XIDADE }) \text {, }
$$

onde XIDADE (= IDADE/ESTCRE) é a idade da cultura (dias após o plantio) relativa à estação de crescimento (ESTCRE), isto é, o período entre o plantio e a colheita. Pode-se interpretar XIDADE como idade fisiológica da cultura 
permitindo comparação entre culturas com ciclos diferentes (12 ou 18 meses). FIDADE $=1,0$ para $X I D A D E \leqslant 0,15$.

Fator índice de área foliar (FIAF) - Ao penetrar o dossel vegetativo, a radiação solar é atenuada em função da área foliar acumulada verticalmente desde o topo da cultura. Resultados experimenta is evidenciam que tal atenuação é, em geral, exponencial (Lei de Beer). Devido ao sombreamento, as folhas inferiores recebem menor quantidade de radiação, havendo redução em sua taxa de fotossíntese. Para compensar tal redução, a cultura aumenta sua área foliar até atingir um máximo. Após esse ponto, há contínua renovação de folhas, sendo as mais velhas substituídas por novas, mais eficientes (HARTT \& BURR, 1965; WALDRON et alii, 1967; GOSNELL, 1968; McLEAN et alii, 1968; MACHADO et alii, 1982). Obteve-se a relação

$$
F I A F=\operatorname{EXP}(0,131-0,14 * I A F),
$$

para descrever o fator índice de área foliar. FIAF $=1,0$ para IAF $\leqslant 1,0$.

Fator temperatura (FTEMP) - Os processos fisiológicos são bastante afetados pela temperatura ambiente. Plantas com metabolismo do tipo C4 apresentam taxa de fotossíntese máxima ao redor de $30-35^{\circ} \mathrm{C}$ (SINGH \& LAL, 1935; EL-SHARKAWY \& HESKETH, 1964; HOFSTRA \& HESKETH, 1969; DOWNTON, 1971; LAAR.\&PENNING de VRIES, 1972; BIRD et alii, 1977), decrescendo rapidamente abaixo de $20^{\circ} \mathrm{C}$. GLOVER (1974) revela que, em cana-de-açúcar, a taxa de fotossíntese apresenta um $Q_{10}$ ao redor de dois entre 15 e $30^{\circ} \mathrm{C}$.

Admitiu-se que a taxa de fotossíntese seja nula abaixo de $12^{\circ} \mathrm{C}$, aumente linearmente até atingir $30^{\circ} \mathrm{C}$, e permaneça constante entre 30 e $35^{\circ} \mathrm{C}$. Portanto,

$$
\text { FTEMP }=0,053 *^{*} \mathrm{~T}-0,59,
$$

onde $T$ é a temperatura, em oC. FTEMP $=1,0$ para $T \geqslant 30 \circ \mathrm{C}$.

Diariamente, a temperatura flutua entre um máximo e um mínimo. Durante o período fotossintetizante, ela está mais próxima do máximo, havendo necessidade de estimar uma temperatura efetiva para a fotossíntese. Contornou-se tal problema, atribuindo-se pesos diferentes para as temperaturas máxima e mínima diária, ou seja,

$$
T=\left(2^{*} T M A X+T M I N\right) / 3 \text {. }
$$

Fator umidade (FUR) - A taxa de fotossíntese é afetada pela condição hídrica da planta, visto que a abertura dos estômatos controla a absorção de $\mathrm{CO}_{2}$. A condição hídrica de uma cultura é governada pelo balanço entre 
a perda de água através da transpiração e o suprimento através das raízes. A perda de água é determinada por muitos fatores, entre os quais se destaca o déficit de saturação atmosférico, ou seja, a demanda atmosférica. DENMEAD \& SHAW (1962) mostram que, se a demanda for baixa, a cultura será capaz de extrair água do solo mesmo sob condições de baixa umidade disponível; por outro lado, se a demanda for alta, a cultura mostrará sintomas de deficiência mesmo com alta umidade disponível. Como existe estreita relação entre fotossíntese e transpiração, é lógico supor que uma redução na taxa de transpiração acarretará redução equivalente na taxa de fotossíntese.

Resultados de TAZAKI et alii (1980) mostram redução na taxa de fotossíntese com aumento no déficit de saturação atmosférico. Esse déficit é função da umidade relativa do ar (UR), isto é, def. sat. $=e_{s} *(1-0,01 * U R)$, onde $e_{s}$ é a tensão de saturação do ar a dada temperatura. Como a umidade relativa varia entre 0 e $100 \%$, optou-se pela relação

$$
F U R=0,01 * U R,
$$

onde UR é a umidade relativa média do dia.

$$
\text { Portanto, Fi }=\text { FRAD * FIDADE * FIAF * FTEMP * FUR. }
$$

\subsection{Respiração de manutenção - EFIC * $M$ * PCUL}

A respiração de manutenção, segundo termo da equação (2), representa a quantidade de energia (material) necessária para a manutenção dos processos fisiológicos da cultura, podendo também ser interpretada como o crescimento necessário para contrabalançar a degradação biológica dos tecidos.

Resultados experimenta is indicam que o coeficiente de manutenção $M\left(g . g^{-1} . d^{-1}\right)$ varia com a temperatura (McCREE, 1970, 1974; THORNLEY \& HESKETH, 1972; PENNING de VRIES, 1975a, b; RYLE et alii, 1976; PENNING de VRIES et alii, 1979; LOPES, 1979) e com a idade da planta (EVANS, 1975; HUNT \& LOOMIS, 1979; ACOCK et alii, 1979; LOPES, 1979), sendo, porém, praticamente independente da condição hídrica (PENNING de VRIES et alii, 1979). Adotou-se a expressão

$$
M=\text { MMAX * MTEMP * MIDADE, }
$$

onde $\operatorname{MMAX}\left(=0,007 \mathrm{~g} \cdot \mathrm{g}^{-1} \cdot \mathrm{d}^{-1}\right)$ é a taxa máxima de respiração, MTEMP é o fator temperatura, e MIDADE, o fator idade da cultura.

Fator temperatura (MTEMP) - Para temperatura entre 20 e $30 \mathrm{C}^{\circ}$, a taxa de respiração em caria-de-açúcar apresenta um $Q_{10}$ ao redor de 2 
(GLOVER, 1973), semelhante ao valor obtido em outras espécies (McCREE, 1970, 1974; PENNING de VRIES, 1975a, b; RYLE et alii, 1976; PENNING de VRIES et alii, 1979; LOPES, 1979).

MCCREE \& SILSBURY (1978) e LOPES (1979) mostram que a taxa de respiração aumenta exponencialmente com a temperatura. Adaptando-se tais resultados, obteve-se a relação

$$
M T E M P=\operatorname{EXP}(0,0607 * \text { TMED }-2,1056),
$$

onde TMED é a tempera tura média diária, isto é, 0,5* (TMAX + TMIN).

Fator idade (MIDADE) - MEDINA et alii (1970) revelam que a taxa de respiração em cana-de-açúcar decresce com a idade da planta. Resultados semelhantes são apresentados em outras espécies (HESKETH et alii, 1971; BAKER et alii, 1972; LOPES, 1979). Adaptando-se os resultados de LOPES (1979), encontrou-se a relação

$$
\operatorname{MIDADE}=\operatorname{EXP}(0,22038-1,34254 * X I D A D E),
$$

onde XIDADE é a idade fisiológica da cultura definida na equação (9). MIDADE $=1,0$ para $X I D A D E \leqslant 0,15$.

\section{PARTIÇÃO DE TCCUL}

O conjunto de equações (2)-(16) permite o cálculo de TCCUL em base diária. Nesse ponto, resta saber como TCCUL se distribui entre os diversos órgãos da planta, ou seja, quanto de TCCUL será utilizado para crescimento de folhas, raízes e colmo. Estimou-se a taxa de crescimento das raízes (TCRAIZ) através da relação

$$
\text { TCRAIZ = FRAIZ * TCCUL, }
$$

sendo que

$$
\text { FRAIZ }=0,9-1,125 * \text { XIDADE. }
$$

A fração raiz (FRAIZ) foi determinada empiricamente e indica que inicialmente a maior parte de TCCUL é util izada na formação das raizes, visando explorar um volume maior de solo e dar sustentação mecânica à parte aérea da planta. FRAIZ decresce linearmente com a idade da cultura até XIDADE = 0,8 ; após essa idade, $F R A I Z=0,0$. Portanto, a taxa de crescimento da parte aérea (TCAER) é dada por

$$
\text { TCAER }=\text { TCCUL }- \text { TCRAIZ. }
$$


Evidentemente, parte de TCAER se transforma em folha (FFOL) e, o restante, em colmo (1-FFOL). MACHADO et alii (1982) mostram que, inic ialmente, as folhas têm preferência sobre o colmo, evidenciando a necessidade de aumentar rapidamente a superfície fotossintetizante, visando à maior captação da energia solar; posteriormente, após o estabelecimento da área foliar, tal tendência é revertida. Adaptando-se tais resultados, obteve-se a expressão

$$
\mathrm{FFOL}=0,95-1,252 * \mathrm{XIDADE},
$$

com as restrições: $F F O L=0,58$ para $X I D A D E \leqslant 0,3$; e $F F O L=0,08$ para $X I D A D E \geqslant 0,9$. Portanto, as taxas de crescimento das folhas (TCFOL) e do colmo (TCCOL) são computadas, respectivamente, através das relações

$$
\begin{aligned}
& \text { TCFOL }=\text { FFOL } * \text { TCAER, } \\
& \text { TCCOL }=(1-F F O L) * \text { TCAER. }
\end{aligned}
$$

Como discutido anteriormente, existe constante renovação de folhas, sendo as mais velhas substituídas por novas, mais eficientes (MACHADO et alii, 1982). Há, portanto, necessidade de introduzir uma taxa de senescência de folhas (TSENF) para evitar que a área foliar cresça continuamente até valores irreais. Devido à falta de informação específica, quantificou-se empiricamente tal taxa através do produto de um fator de senescência (FSEN), que varia com a idade da cultura, e da taxa de crescimento das folhas, ou seja,

$$
\begin{aligned}
& \text { TSENF }=\text { FSEN *TCFOL, } \\
& \text { FSEN }=-0,444+2,222 * \text { XIDADE, }
\end{aligned}
$$

com as restrições: FSEN $=0,0$ se XIDADE $\leqslant 0,20$; e FSEN $=1,0$ se XIDADE $\geqslant 0,65$. No entanto, FSEN deve ser maior que 1,0 para permitir que IAF decresça mesmo com TCCUL positiva. Para tanto, multiplica-se FSEN por FLUZ $^{-1}$ quando o fotoperiodo for menor que 12 horas.

Concomitantemente, deve haver também uma taxa de senescência das raízes (TSENR). Aqui, a falta de informações é maior, visto que as raízes crescem num meio opaco e de difícil acesso. Na presente versão, adotou-se a seguinte aproximação.

$$
\text { TSENR }=\text { CONST } * \text { FSEN * TCRAIZ, }
$$

onde CONST $=0,5$ e FSEN é o mesmo fator utilizado para senescência das folhas. Assumiu-se, portanto, que a taxa de senescência das raízes seja a metade da taxa de senescência das folhas. 


\section{CRESCIMENTO DA CULTURA}

Num dado dia t, a massa seca de cada parte da cultura será dada por

$$
\begin{aligned}
& \text { PRAIZ }(t)=\text { PRAIZ }(t-1)+\text { TCRAIZ }- \text { TSENR, } \\
& \text { PFOL }(t)=\text { PFOL }(t-1)+\text { TCFOL }- \text { TSENF, } \\
& \text { PCOL }(t)=\text { PCOL }(t-1)+\text { TCCOL. }
\end{aligned}
$$

Conseqüentemente, $\operatorname{PCUL}(t)$ é dada pela soma das equações $(26),(27)$ e (28).

$O$ índice de área foliar (IAF) é obtido através do produto da massa seca das folhas (PFOL) com área foliar específica (AFE), isto é,

$$
I A F=P F O L * A F E .
$$

MACHADO et alii (1982) indicam que AFE é aproximadamente igual a $1 \mathrm{dm}^{2} \cdot \mathrm{g}^{-1}$, e praticamente independente da idade da cultura.

\section{VERIFICAÇÃO E VALIDAÇÃO DE SIMCANA}

Como SIMCANA não simula o processo de germinação, há necessidade de definir as condições iniciais da cultura para o primeiro dia de simulação, ou seja, a massa seca por unidade de área do colmo (PCOL), das folhas (PFOL), das raízes (PRAIZ) e o índice de área foliar (IAF), bem como a estação de crescimento (ESTCRE, em dias), a idade da cultura (IDADE, em dias após o plantio), o número do dia no ano do primeiro dia de simulação (a saber: e.g., 1 ọ de fevereiro corresponde a $D \backslash A=32$ ), e a latitude do local (ALATI, em graus e décimos).

\section{PIRACICABA, 1978/79}

Para verificar o comportamento de SIMCANA, utilizaram-se os dados da análise de crescimento das variedade NA56-79 e CB41-14 obtidos por MACHADO et alii (1982) na Estação Experimental de Piracicaba, SP ( $\left.A L A T I=-22,3^{\circ}\right)$, do Instituto Agronômico de Campinas. Tal experimento foi plantado em março de 1978 e colhido em junho de 1979, tendo uma estação de crescimento (ESTCRE) de aproximadamente 450 dias. A metodologia utilizada na obtenção dos dados, e as condições do experimento, estão descritas em MACHADO et alii (1982). Os dados meteorológicos diários necessários à execução do modelo, isto é, radiação solar (RAD), tempe- 
ratura máxima (TMAX) e mínima (TMIN), e umidade relativa do ar (UR), foram obtidos no posto meteorológico situado numa área adjacente ao experimento.

A simulação iniciou-se em 8 de agosto, que corresponde a $D \perp A=$ $=.220$ e IDADE $=133$ dias. As condições jniciais da cultura foram: $P C O L=$ $=80 \mathrm{~g} ; \mathrm{PFOL}=100 \mathrm{~g} ; \mathrm{PRAIZ}=27 \mathrm{~g} ; \mid \mathrm{AF}=1,0$.

A figura 1 mostra a variação de IAF e da massa seca da parte aérea das culturas entre 130 e 430 dias após o plantio. Nota-se que SIMCANA simulou bem tanto IAF como a massa seca durante o período considerado. Isso, no entanto, já era de esperar, visto que, como explicado anteriormente, muitas das informações obtidas por MACHADO et alii (1982) foram utilizadas no desenvolvimento de SIMCANA. Não se pode admitir, portanto, que os dados desse experimento sejam considerados como teste real do desempenho de SIMCANA. Aproveitou-se de tal fato, todavia, para realizar, com base nos resultados da simulação, alguns ajustes (calibragem) nas relações empíricas desenvolvidas sem respaldo experimental. Esse é um processo iterativo bastante prolongado e subjetivo, visto que as funções utilizadas não são lineares e têm efeito multiplicativo no processo envolvido. Verifica-se, no entanto, em função da resposta final do modelo, que as relações empíricas adotadas principalmente para o fator umidade (FUR), taxa de crescimento das raízes (TCRAIZ), e taxas de senescência das folhas (TSENF) e das raízes (TSENR) não estão longe da realidade.

\section{ARARAS, $1981 / 82$}

Para testar realmente a validade de SIMCANA (validação do modelo), há, portanto, necessidade de um conjunto independente de dados que não tenham sido sequer utilizados para ajuste das diversas funções desenvolvidas.

Visando obter tal conjunto de dados, foi instalado um experimento na Estação Experimental do Planalsucar - IAA, em Araras, SP, em outubro de 1981. Utilizaram-se também duas variedades, NA56-79 e CB47-355. Adotou-se a mesma metodologia descrita por MACHADO et alii (1982) para a obtenção de IAF e massa seca da parte aérea da cultura. Os dados meteorológicos foram obtidos no posto meteorológico situado a menos de $200 \mathrm{~m}$ da área experimental. O experimento foi colhido em fins de setembro de 1982, apresentando, portanto, uma estação de crescimento (ESTCRE) de aproximadamente 350 dias.

As cond ições inic iais de simulação foram: $\mathrm{PCOL}=2,7 \mathrm{~g} ; \mathrm{PFOL}=8,0 \mathrm{~g}$; PRAIZ =6,0g; IAF =0,08; DIA = 348 (15 de dezembro); e, IDADE = $=61$ dias. 


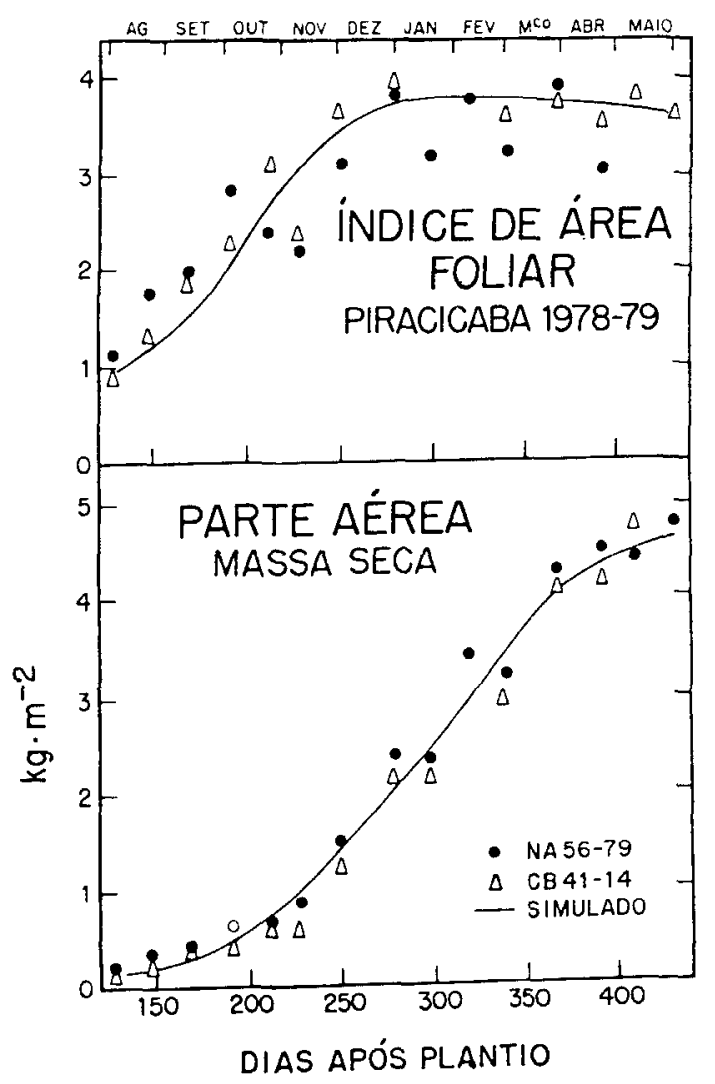

FIGURA 1. Massa seca da parte aérea e índice de área foliar em duas culturas de cana-de-açucar. Piracicaba, SP, $1978 / 79$.

A figura 2 mostra que SIMCANA simulou IAF adequadamente também nas condições de Araras. A grande dispersão dos pontos evidencia a dificuldade dessas medidas em condições de campo. O IAF máximo obtido em Araras foi aproximadamente metade daquele observado em Piracicaba. Tal redução em IAF causou uma redução ainda maior na massa seca da parte aérea. Esse experimento mostra claramente o efeito do fotoperíodo sobre o crescimento da cana-de-açúcar. O início do desenvolvimento de IAF 
coincidiu com a época em que o fotoperíodo começou a decrescer. Nota-se que o IAF cresceu somente até o fotoperíodo atingir aproximadamente 12 horas (21 de março).

Entre 200 e 300 dias após o plantio, o cv. NA56-79 apresentou maior massa seca da parte aérea. No entanto, ao final do ciclo (350 dias), tal diferença praticamente desapareceu, evidenciando certa precocidade desse cultivar em relação ao CB47-355. No mesmo intervalo de tempo (200300 d.a.p.), SIMCANA simulou uma condição mais próxima da massa seca do cv. CB47-355.

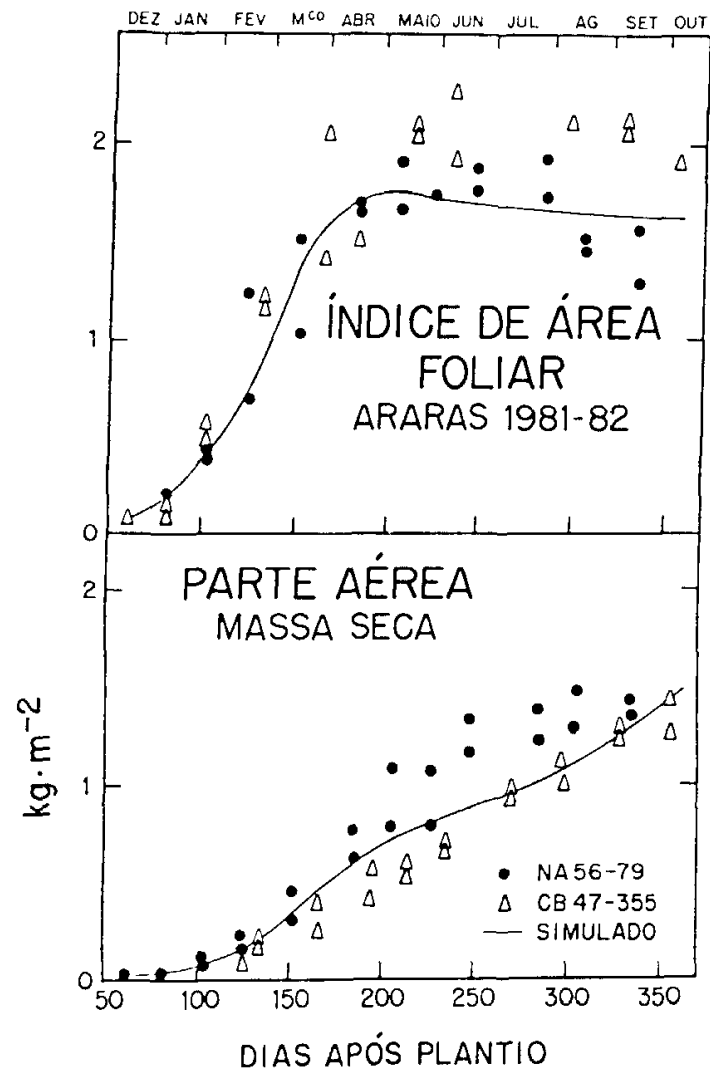

FIGURA 2. Massa seca da parte aérea e indice de área foliar em duas culturas de cana-de-açúcar. Araras, SP, 1981/82. 


\section{DISCUSSĀO}

E preciso, de início, reconhecer que o modelo SIMCANA é, em mu itos aspectos, deficiente e incompleto. No entanto, parece resumir adequadamente as informações disponiveis concernentes aos principais processos fisiológicos da cultura de canade-açúcar.

As deficiências do modelo devem-se principalmente à inexistência de informações específicas passíveis de ser quantificadas. Para cobrir tais acunas da literatura, optou-se pela utilização de relações empíricas baseadas apenas na intuição que seus autores têm do crescimento da cultura. Evidentemente, tais relacões devem ser questionadas com a execução de experimentos específicos, visto que a criação de novos dados e evidências é prioridade única da experimentação. Obviamente, poder-se-ia continuar o processo de calibragem do modelo visando melhorar as relações empíricas que, no entanto, continuariam empíricas.

SIMCANA é incompleto por não considerar vários aspectos, tais como nutrição e sanidade, que invariavelmente afetam o crescimento de uma cultura. O sucesso de uma primeira aproximação evidentemente estimulará a tentativa de incorporação de tais aspectos no modelo.

Note-se, no entanto, que embora várias relações empíricas tenham sido usadas, SIMCANA parece, em sua primeira versão, ser capaz de simular razoavelmente bem o crescimento da cultura de cana-de-açúcar em condições de campo, tanto no ciclo de 12 como no de 18 meses.

SIMCANA utiliza relações simples, não sendo necessária a utilização de máquinas sofisticadas para sua execução. Sua melhoria, isto é, sua universalização, dependerá da utilização de novos dados experimentais obtidos também com outras variedades e em outras condições de ambiente.

\section{AGRADECIMENTOS}

Os autores agradecem aos pesquisadores Valter Barbieri e Udo Rosenfeld, da Seção de Irrigação e Climatologia do Planalsucar - IAA, a œssão dos dados referentes ao experimento de Ararà.

\section{SUMMARY}

\section{A DYNAMIC SIMULATOR OF THE SUGARCANE CROP GROWTH}

The first version of a mathematical-physiological simulator of the daily growth of a sugarcane crop (SIMCANA) as a function of the 
environmental conditions during the growing season is described. SIMCANA summarizes most of the available information regarding to the physiological processes of the sugarcane crop. This version does not include the germination and flowering processes, therefore it is necessary to specify the crop conditions at the first day of simulation. Given the daily conditions of global solar radiation, maximum and minimum temperature, and the relative humidity, SIMCANA computes the rates of crop photosynthesis, respiration, and growth, the senescence rates for leaves and roots, the dry mass of leaves, stems, and roots, and the leaf area index. Although several empirical relations have been used, SIMCANA seems to be able to simulate the sugarcane crop growth.

Index terms: simulation, mathematical-physiological model, photosynthesis rate, respiration rate, growth rate, leaf area index, sugarcane.

\section{REFERÊNCIAS BIBLIOGRÁFICAS}

ACOCK, B.; CHARLES-EDWARDS, D.A. \& SAWYER, S. Growth response of a chrysanthemum crop to the environment. III - Effects of radiation and temperature on dry matter partitioning and photosynthesis. Annals of Botany, 44:289-300, 1979.

BAKER, D.N.; HESKETH, J.D. \& DUNCAN, W.G. Simulation of growth and yield in cotton: I. Gross photosynthesis, respiration, and growth. Crop Science, 12:431-435, 1972.

BIRD, I.F.; CORNELIUS, M.J. \& KEYS, A.J. Effects of temperature on photosynthesis by maize and wheat. Journal of Experimental Botany, 28:519-524, 1977.

BULL, T.A. The C4 pathway related to growth rates in sugarcane. In: HATCH, M.D.; OSMOND, C.B. \& SLATYER, R.O., eds. Photosynthesis and photorespiration. Canberra, John Wiley, 1971. p.68-75.

- Photosynthesis efficiencies and photorespiration in Calvin cycle and C4-dicarboxylic acid plants. Crop Science, 9:726-729, 1969.

\& TOVEY, D.A. Aspects of modelling sugarcane growth by computer simulation. Proceedings of the International Society of Sugar Cane Technologists, 15:1021-1032, 1974.

DENMEAD, O.T. \& SHAW, R.H. Availability of soil water to plants as affected by soil moisture content and meteorological conditions. Agronomy Journal, 45:385-390, 1962.

DOWNTON, W.J.S. Adaptive and evolutionary aspects of $\mathrm{C} 4$ photosynthesis. In: HATCH, M.D.; OSMOND, C.B. \& SLATYER, R.O., eds. Photosynthesis and photorespiration. Canberra, John Wiley, 1971. p.3-17.

EL-SHARKAWY, M. \& HESKETH, J.D. Effects of temperature and water deficit on leaf photosynthesis rates of different species. Crop Science, 4:514-518, 1964.

EVANS, L.T. Beyond photosynthesis - the role of respiration, translocation and growth potential in determining productivity. In: COOPER, J.P., ed. Photosynthesis and productivity in different environments. London, Cambridge Univ. Press, 1975. p.501-507.

GLOVER, J. The dark respiration of sugar-cane and the loss of photosynthate during the growth of a crop. Annals of Botany, 37:845-852, 1973.

. The rate of apparent photosynthesis of whole sugarcane plants. Annals of Botany, 38:909-920, 1974. 
GOSNELL, J.M. Some effects of increasing age on sugarcane growth. Proceedings of the International Society of Sugar Cane Technologists, 13:499-513, 1968.

GOUDRIAAN, J. \& LAAR, H.H. van. Calculation of daily totals of the gross $\mathrm{CO}_{2}$ assimilation of leaf canopies. Netherlands Journal of Agricultural Science, 26:373-382, 1978.

HARTT, C.E. \& BURR, G.O. Factors affecting photosynthesis in sugar cane. Proceedings of the International Society of Sugar Cane Technologists, 12:590-609, 1965.

HESKETH, J.D.; BAKER, D.N. \& DUNCAN, W.G. Simulation of growth and yield in cotton: respiration and carbon balance. Crop Science, 11:394-398, 1971.

HODGES, T.; KANEMASU, E.T. \& TEARE, I.D. Modeling dry matter accumulation and yield of grain sorghum. Canadian Journal of Plant Science, 59:803-818, 1979.

HOFSTRA, G. \& HESKETH, J.D. Effects of temperature on the gas exchange of leaves in the light and dark. Planta, 85:288-297, 1969.

HOLT, D.A.; BULA, R.J.; MILES, G.E.; SCHREIBER, M.M. \& PEART, R.M. Environmental, physiology, modelling and simulations of alfalfa growth. I - Conceptual development of SIMED. West Lafayette, Ind., Purdue University, Agricultural Experiment Station, 1975. (Res. Bull. 905)

HUNT, W.F. \& LOOMIS, R.S. Respiration modelling and hypothesis testing with a dynamic model of sugar beet growth. Annals of Botany, 44:5-17, 1979.

IRVINE, J. Sugarcane. In: SYMPOSIUM ON POTENTIAL PRODUCTIVITY OF FIELD CROPS UNDER DIFFERENT ENVIRONMENTS. Los Baños, Philippines, IRRI, 1983. p.361-381.

JAGER, J.M. 'PUTU' a dynamic seasonal maize crop growth model. In: GUELPH Project, Final Report, Ontario, Univ. Guelph, 1974. p.306-320.

- \& KING, K.M. Calculation of photosynthesis rate of a maize crop from environmental variables. In: GUELPH Project, Final Report, Ontario, Univ. Guelph, 1974. p.321-340.

KORTSCHAK, H.P. \& FORBES, A. The effects of shade and age on the photosynthesis rate of sugarcane. In: METZNER, $H_{\text {, }}$ ed. Progress in photosynthesis research. Tübingen, 1969 . v.1, p.383-387.

LAAR, H.H. van \& PENNING de VRIES, F.W.T. $\mathrm{CO}_{2}$-assimilation light response curves of leaves; some experimental data. Wageningen, Versl. Inst. Biol. Scheik Onderz. LandbGewassem 62, 1972.

LOPES, N.F. Respiration related to growth and maintenance in radish (Raphanus sativus L.) plants. Davis, University of California, 1979. 151p. Thesis.(Ph.D.)

MACHADO, E.C.; PEREIRA, A.R.; FAHL, J.I.; ARRUDA, H.V. \& CIONE, J. Índices biométricos de duas variedades de cana-de-açúcar. Pesquisa Agropecuária Brasileira, Brasilia, 17:1323-1329, 1982.

- - - - \& CAMARGO, M.B.P. Relaçōes radiométricas de uma cultura de cana-de-açúcar. Bragantia, Campinas, 44(1):229-238, 1985.

McCREE, K.J. An equation for the rate of respiration of white clover plants grown under controlled conditions. In: PREDICTION and measurement of photosynthetic productivity. Wageningen, PUDOC, 1970. p.221-229.

Equations for the rate of dark respiration of white clover and grain sorghum, as functions of dry weight, photosynthetic rate, and temperature. Crop Science, 14:509-514, 1974. 
McCREE, K.J. \& KRESOVICH, S. Growth and maintenance requirements of white clover as a function of daylength. Crop Science, 18:22-25, 1978.

\& SILSBURY, J.H. Growth and maintenance requirements of subterranean clover. Crop Science, 18:13-18, 1978.

McLEAN, F.G.; McDAVID, C.R. \& SINGH, Y. Preliminary results of net assimilation rate studies in sugarcane. Proceedings of the International Society of Sugar Cane Technologists, 13:849-858, 1968.

MEDINA, E.; SAN JOSÉ, J.J. \& SEQUERA, P.E. Análisis de la productividad en caña de azúcar. III - Respiración en la obscuridad de hojas y tallos de cinco variedades de caña de azúcar y pérdidas nocturnas de materia seca. Turrialba, 20:302-306, 1970.

PENNING de VRIES, F.W.T. The cost of maintenance processes in plant cells. Annals of Botany, 39:77-92, 1975b.

_- Use of assimilates in higher plants. In: COOPER, J.P., ed. Photosynthesis and productivity in different environment. London, Cambridge Univ. Press, 1975a. p.459-480.

-; WITLAGE, J.M. \& KREMER, D. Rates of respiration and of increase in structural dry matter in young wheat, ryegrass and maize plants in relation to temperature, to water stress and to their sugar content. Annals of Botany, 44:595-609, 1979.

PEREIRA, A.R.; BARBIERI, V. \& VILLA NOVA, N.A. Climatic conditioning of flowering in sugarcane. Agricultural Meteorology, 29:103-110, 1983.

RYLE, G.J.A.; COBBY, J.M. \& POWELL, C.E. Synthetic and maintenance respiratory losses of ${ }^{14} \mathrm{CO}_{2}$ in uniculm barley and maize. Annals of Botany, 40:571-586, 1976.

SINGH, B.N. \& LAL, K.N. Limitations of Blackman's law of limiting factors and Harder's concept of relative minimum as applied to photosynthesis. Plant Physiology, 10:245-268, 1935.

TAZAKI, T.; ISHIHARA, K. \& USHIJIMA, T. Influence of water stress on the photosynthesis and productivity of plants in humid areas. In: TURNER, N.C. \& KRAMER, P.J., eds. Adaptation of plants to water and high temperature stress. New York, Wiley-Interscience, 1980. p.309-321.

THORNLEY, J.H.M. Mathematical models in plant physiology: a quantitative approach to problems in plant and crop physiology. London, Academic Press, 1976. 318p.

- Respiration, growth and maintenance in plants. Nature, 227:304-305, 1970.

\& HESKETH, J.D. Growth and respiration in cotton bolls. Journal of Applied Ecology, 9:315-317, 1972.

VALSECHI, O. \& OLIVEIRA, E.N. A cana-de-açúcar como materia-prima. In: CULTURA e adubação da cana-de-açúcar. São Paulo, Instituto Brasileiro de Potassa, 1964. p.319-368.

WALDRON, J.C.; GLASZIOU, K.T. \& BULL, T.A. The physiology of sugar cane. IX Factors affecting photosynthesis and sugar storage. Australian Journal of Biological Sciences, 20:1043-1052, 1967.

WILSON, D.R.; BAVEL, C.H.M. van \& McCREE, K.J. Carbon balance of water-deficient grain sorghum plants. Crop Science, 20:153-159, 1980.

WHIT, C.T. de; LAAR, H.H. van \& KEULEN, H. van. Physiological potential of crop production. In: SNEEP, J. \& HENDRIKSEN, A.J.T., eds. Plant breeding perspective. Wageningen, PUDOC, 1979. p.47-82. 